\title{
The first generations of stars
}

\author{
Elisabetta Caffau ${ }^{* a, b}$, A. J. Gallagher ${ }^{a}$, P. Bonifacio ${ }^{a}$, R. Cayrel ${ }^{a}$, N. Christlieb ${ }^{b}$, P. C. \\ Clark $^{c, d}$, P. François ${ }^{a}$, S. Glover ${ }^{d}$, R.S. Klessen ${ }^{d}$, A. Koch ${ }^{b}$, H.-G. Ludwig ${ }^{b, a}$, L. Monaco ${ }^{e}$, \\ B. Plez ${ }^{f}$, L. Sbordone ${ }^{g, h, b}$, M. Spite ${ }^{a}$, F. Spite ${ }^{a}$, M. Steffen $^{i, a}$, S. Zaggia ${ }^{j}$ \\ ${ }^{a}$ GEPI, Observatoire de Paris, CNRS, Univ. Paris Diderot, Place Jules Janssen, 92195 Meudon, \\ France \\ ${ }^{b}$ Zentrum für Astronomie der Universität Heidelberg, Landessternwarte, Königstuhl 12, 69117 \\ Heidelberg, Germany \\ ${ }^{c}$ School of Physics and Astronomy, The Parade, Cardiff University, Cardiff, CF24 3AA \\ ${ }^{d}$ Zentrum für Astronomie der Universität Heidelberg, Institut für Theoretische Astrophysik, \\ Albert-Ueberle-Straße 2, 69120 Heidelberg, Germany \\ ${ }^{e}$ European Southern Observatory, Casilla 19001, Santiago, Chile \\ ${ }^{f}$ Laboratoire Univers et Particules de Montpellier, LUPM, Université Montpellier 2, CNRS, \\ 34095 Montpellier cedex 5, France \\ ${ }^{g}$ Millennium Institute of Astrophysics, Vicuña MacKenna 4860, Macul, Santiago, Chile \\ ${ }^{h}$ Pontificia Universidad Católica de Chile Vicuña MacKenna 4860, Macul, Santiago, Chile \\ ${ }^{i}$ Leibniz-Institut für Astrophysik Potsdam (AIP), An der Sternwarte 16, 14482 Potsdam, \\ Germany \\ ${ }^{j}$ Istituto Nazionale di Astrofisica, Osservatorio Astronomico di Padova Vicolo dell'Osservatorio \\ 5, 35122 Padova, Italy
}

Extremely metal-poor (EMP) stars are descendants of massive stars from the earliest stellar generations. Their chemical composition reflects the early phases of the Universe, which allows us to place constraints on the masses of these first-generation massive stars and also allows us to better understand the formation of low-mass stars from a gas cloud depleted in metals. EMP stars are a minor component of the stellar population (about a thousandth) and special care has to be invested to find them. We exploited the Sloan Digital Sky Survey (SDSS) to search for EMP candidates with the colours of Turn-Off stars. We observed them at medium- and high-resolution with the ESO/VLT spectrographs X-Shooter and UVES to derive their chemical composition. We present the preliminary results on the metallicity distribution function, on the analysis of strontium, on the carbon enhanced stars, and on our effort to analyse molecular bands with 3D hydrodynamical models.

XIII Nuclei in the Cosmos,

7-11 July, 2014

Debrecen, Hungary

\footnotetext{
* Speaker.

$\dagger$ The project has been financed by FOUNDATION MERAC.
} 


\section{Introduction}

The very early Universe was composed of hydrogen, helium, and traces of lithium. Therefore, the first stars that formed should have had this composition. Metals are thought to play an essential role in cooling the collapsing molecular cloud these stars would have formed from, and the absence of metals would result in the formation of a relatively massive first generation of stars, but this is a purely theoretical paradigm requiring observational confirmation. In fact, we know that a gas that contracts under a gravitational force increases in temperature, and the extra heat has to be dispersed to allow the formation of stars, otherwise the thermal pressure due to the kinetic energy of the gas particles will balance the gravitational force, and no star will form. We know that to cool a gas, metals are an efficient agent through collisional excitation followed by radiative de-excitation. If the mass of a gas under contraction is large the gravitational force is large, no balance with the thermal pressure is reached because the transitions of the hydrogen atoms are sufficient to disperse the extra energy, and a high-mass star (tens of solar masses or more) can form. The first stellar generation is therefore likely to be dominated by high-mass stars, although the formation of at least a few low-mass stars cannot be ruled out (Bromm, 2013; Glover, 2013).

The first generation massive stars evolved rapidly, with lifetimes of the order of several Myr. A $30 \mathrm{M}_{\odot}$ star, for instance, has a lifetime of $5.8 \mathrm{Myr}$, while the lifetime of a star of $60 \mathrm{M}_{\odot}$ is $3.6 \mathrm{Myr}$ (Limongi \& Chieffi, 2006). The larger the stellar mass, the higher the temperature in its core, therefore the burning of hydrogen becomes more efficient and hydrogen lasts for a shorter amount of time in the stellar core. During their short lives these massive stars synthesised metals in their interior, that were ejected into the interstellar medium as they exploded as supernovae at the end of their life, enriching the gas with the elements synthesised. Only a fraction of the mass of the star, the remnant, is left as a neutron star or a black hole.

The second generation of stars formed from gas enriched in metals. Simulations suggest that the metal enrichment greatly promotes the formation of low-mass stars (see e.g. Dopcke et al., 2013), and indeed the oldest stars that we have discovered so far date from this epoch. They are the oldest objects in the Galaxy, formed within the first billion years after the big bang (corresponding to a redshift of $z>4$ ), and their chemical composition reflects the early enrichment of the matter by the first massive stars, which after reionising the Universe died as supernovae. We are particularly interested in these low mass $\left(M<\mathrm{M}_{\odot}\right)$ stars because their lifetime is of the order of $13 \mathrm{Gyr}$, so a low-mass star that formed in the early phases of the Universe is still shining today in the sky. The Sun has a main sequence lifetime of $10 \mathrm{Gyr}$, while a star of smaller mass, $0.8 \mathrm{M}_{\odot}$, has a lifetime of 12 - 13 Gyr; a time comparable to the age of the Universe.

A plethora of complex chemical elements, from carbon to uranium, present in stars, were synthesised by stars of previous generations. Successive generations of stars formed out of material which was progressively more chemically enriched. Astronomers have been striving to find stars as metal-poor as possible in order to understand the very first steps of the build-up of the chemical elements, and even to find stars with the metal-free primordial composition, if such stars still exist.

We have been involved in the search and chemical analysis of extremely metal-poor (EMP) stars (see e.g. Bonifacio et al., 2012; Caffau et al., 2013) for several years. Here, we briefly present some recent results and work in progress. 


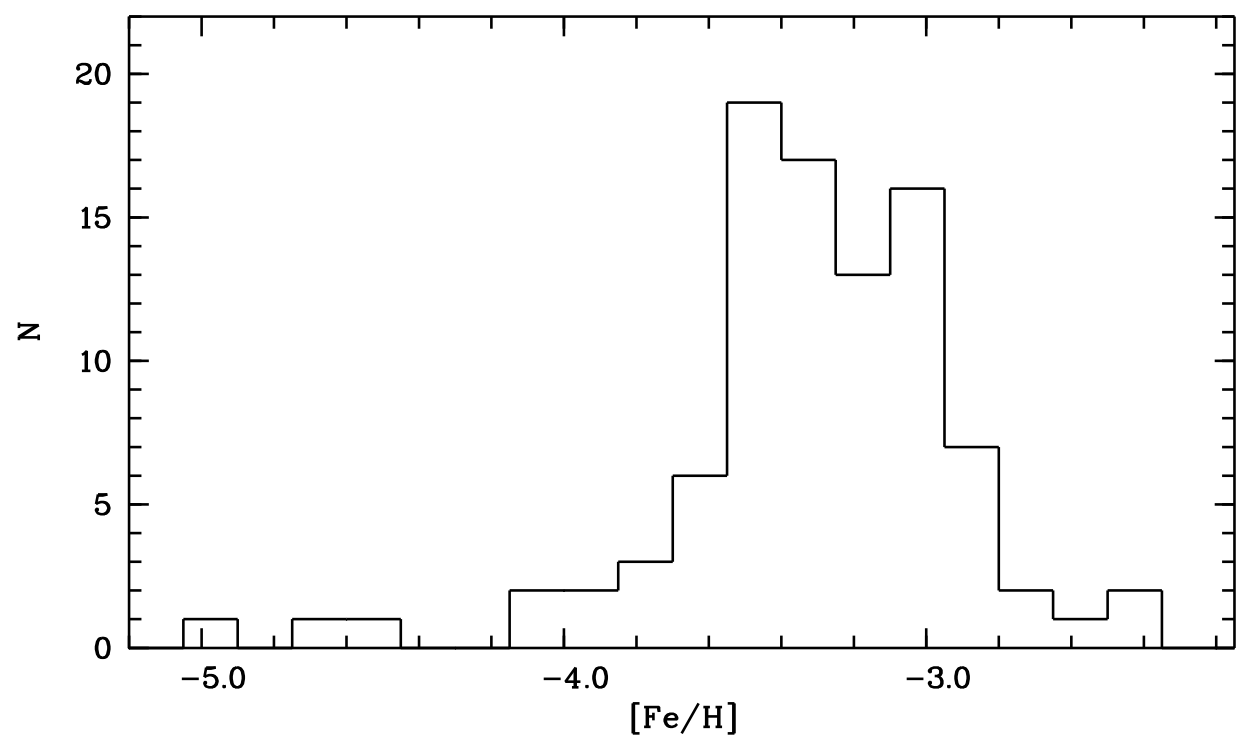

Figure 1: The MDF derived from the sample of 96 SDSS stars we observed at high- and medium-resolution. The metallicity is derived from the high- and medium-resolution spectra. The work is still in progress.

\section{Metallicity Distribution Function}

By improving our understanding of the low metallicity end of the metallicity distribution function (MDF), we can test which theoretical models of star formation agree with the data. We selected about 180000 spectra of turn-off (TO) stars in the Sloan Digital Sky Survey (SDSS) (York et al., 2000; Yanny et al., 2009; Dawson et al., 2013). The metallicity we derive with our pipeline from these spectra provides us with representative statistics of the metallicity distribution function. The spectra of SDSS have a low-resolution (1 800 in the blue arm for releases up to DR8, 1400 for DR9) and the chemical abundance determination is affected by a large uncertainty (about 0.5 dex). In the case of the metal-poor end of the MDF, the spectra show very few features, and any metallic lines are usually very weak. For the most metal-poor stars (especially in the case of the hottest F-stars), only the Ca II-K line is clearly measurable. Calibration of the metallicity of a sub-sample of these EMP stars can allow us to reduce, if not remove, systematic uncertainties in their metallicity. With a sample of 96 stars observed at high- and medium-resolution (UVES and X-Shooter) we will calibrate the chemical analysis of the SDSS sample. In Fig. 1 the MDF of our sample of EMP stars, selected from the SDSS, is shown.

The vertical drop in the metal-poor end of the MDF shown in Schörck et al. (2009) at $[\mathrm{M} / \mathrm{H}]=$ -3.5 , revealing a "critical metallicity" required for the formation of low mass stars is not visible in this sample. Several stars we analysed have an iron abundance of $[\mathrm{Fe} / \mathrm{H}]<-3.5$. In Fig. 2 the MDF of the sample of our stars is compared to the MDF based on our analysis of the SDSS sample observable from Paranal and brighter than $g<19.5$. With this figure we wish to show the large number of expected EMP stars from the low resolution SDSS spectra. With the medium- and high-resolution observations we will calibrate the low-resolution observations in order to reduce the number of "false-positives". 


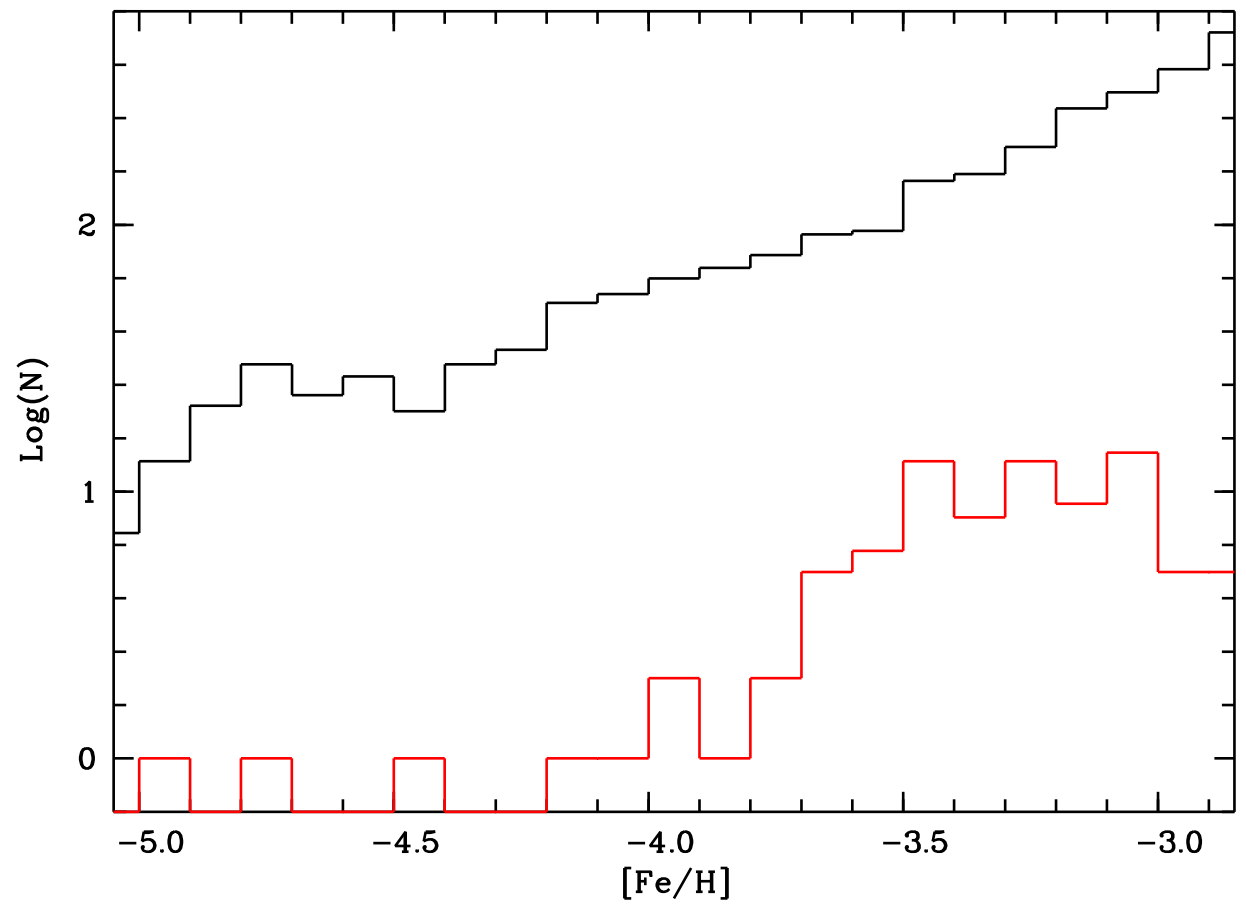

Figure 2: The MDF of our sample of 96 stars (solid red) compared to the SDSS stars (solid black) observable from Paranal $\left(\mathrm{dec}<28^{\circ}\right)$ and bright enough to be potentially observable with X-Shooter $(\mathrm{g}<19.5)$.

\section{Strontium abundance}

Strontium is a key element to probe the chemical evolution in the early stages of the Galaxy. In metal-poor regimes, it is produced by a charged particle process or by the slow (s-) neutron-capture process in rapidly rotating massive stars (see e.g. Hansen et al., 2013, and references therein). This is the only heavy element (sometimes with barium) that can be detected in unevolved EMP stars. Two Sr II line are present in the X-Shooter range, at 407.7 and $421.5 \mathrm{~nm}$. In Fig. 3 the preliminary strontium abundance for our sample of stars is shown. The strontium abundance in old stars is not uniform, showing a large star-to-star scatter in $[\mathrm{Sr} / \mathrm{Fe}]$ even when taking into account departures from local thermodynamical equilibrium (Andrievsky et al., 2011). Our sample of stars behaves similarly to the sample of Andrievsky et al. (2011) with only one star with a high strontium abundance.

\section{Carbon enhanced metal-poor stars}

Carbon-enhanced extremely metal-poor (CEMP) stars could be classified as peculiar objects. Their chemical pattern is different from "normal" EMP stars. In a carbon-normal EMP star, the abundance ratios of the elements with respect to iron are, by and large, similar to what we find in the Sun, with a few noticeable exceptions, like the $\alpha$-elements that are slightly enhanced with respect to iron, $([\alpha-$ element $/ \mathrm{Fe}] \sim+0.4)$, sodium that is underabundant, zinc that is over-abundant and so on. Vice-versa, all CEMP stars are characterised by a large overabundance of carbon $([\mathrm{C} / \mathrm{Fe}]>$ +1.0 dex), very often a similar over-abundance of nitrogen and oxygen, sometimes magnesium as 


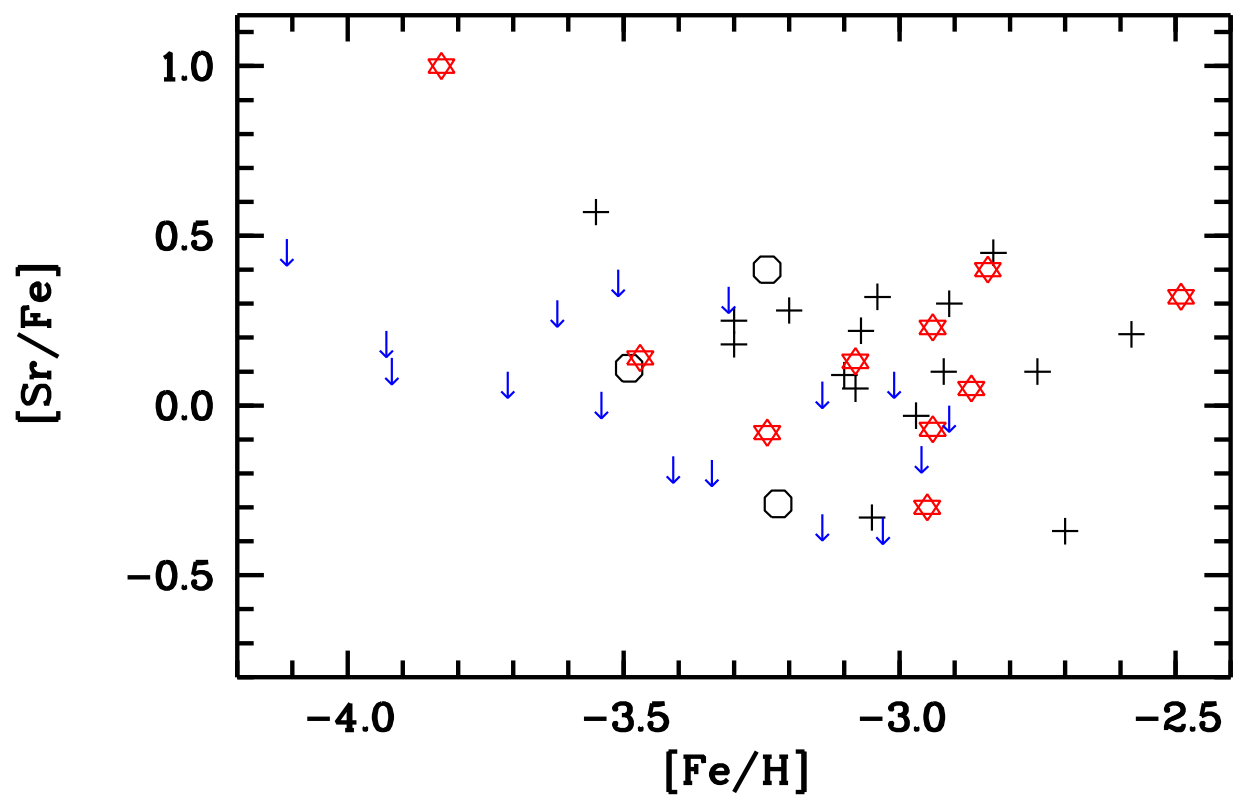

Figure 3: $[\mathrm{Sr} / \mathrm{Fe}]$ versus $[\mathrm{Fe} / \mathrm{H}]$ for the TOPoS (Caffau et al., 2013) stars (red stars) with derived abundance. The blue downward arrows represent the upper limits for a subsample of the stars (the work is still in progress). As a comparison the sample of Andrievsky et al. (2011) (black crosses) and the three stars from Caffau et al. (2011a) (black circles) are also plotted. All measurements take into account departs from local thermodynamical equilibrium.

well, and they show a notable star-to-star scatter in the abundances of the heavy elements. CEMP stars can be enriched in heavy elements built by both the slow (s-) and the rapid (r-) neutroncapture processes, in which case they are called CEMP-rs stars; if they are enriched only in heavy elements built by the s-process they are denominated CEMP-s. CEMP stars with a normal pattern of the heavy elements are called CEMP-no stars. According to Masseron et al. (2010) CEMP$s$ and CEMP-rs show a chemical pattern that suggests mass-transfer from a companion on the asymptotic giant branch (AGB). The large fraction of CEMP-s and CEMP-rs stars with variation in radial velocity led Lucatello et al. (2005) to suggest that they are all binaries, as later confirmed by Starkenburg et al. (2014).

From theory we know that three mechanisms can explain the formation of low-mass stars from a metal-poor gas:

- A low-mass star can form from a zero-metal gas through disk fragmentation (Clark et al. 2011). However, no star of this type has yet been observed.

- $\mathrm{O}$ I and $\mathrm{C}$ Ir fine-structure transitions cooling through collisional excitation and radiative deexcitation (Bromm \& Loeb, 2003). A minimum amount of oxygen and carbon (critical metallicity) is necessary for the star formation. This theory can explain the formation of the first extremely iron-poor carbon-enhanced (CEMP) stars found (Christlieb et al. 2002; Frebel et al. 2005; Norris et al. 2007) and the more recently discovered stars (Keller et al., 2014; Hansen et al., 2014; Bonifacio et al., 2015).

- Dust cooling and fragmentation theory (Schneider et al., 2006; Omukai et al., 2008; Dopcke et al., 2011; Schneider et al., 2012; Dopcke et al., 2013). This requires a metallicity larger than a critical value to form low-mass stars, but the critical metallicity is lower than in the 


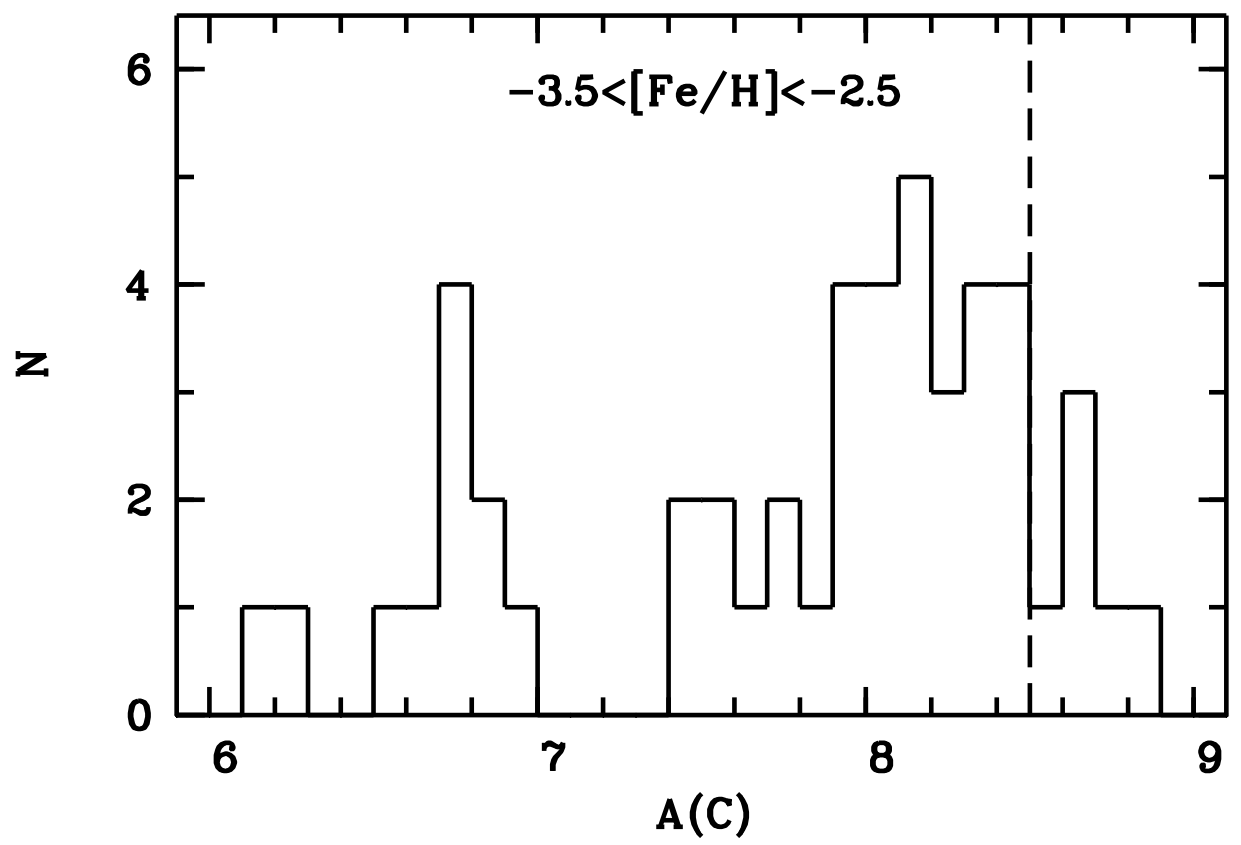

Figure 4: The histogram of the absolute carbon abundance, $\mathrm{A}(\mathrm{C})$, for stars with $-3.5<[\mathrm{Fe} / \mathrm{H}]<-2.5$. The solar carbon is represented by the vertical dashed line. The two $\mathrm{A}(\mathrm{C})$ regions are well separated.

previous case. The formation of SDSS J102915+172927 (Caffau et al., 2011b) can be explained by this theory.

We are investigating CEMP stars to try to better understand star formation. Of the eight stars with $[\mathrm{Fe} / \mathrm{H}]<-4.5$ known to date (Christlieb et al., 2004; Frebel et al., 2005; Keller et al., 2014; Hansen et al., 2014; Bonifacio et al., 2015; Caffau et al., 2011b), seven are CEMP stars. With such a large fraction of CEMP stars in the metal-poor regime, we could be pushed to conclude that they are probably among the oldest stars in the Universe. In Spite et al. (2013) we argued that CEMP stars can be grouped in two regions, according to their carbon abundance:

- The upper band, with an absolute $\mathrm{C}$ abunance, $\mathrm{A}(\mathrm{C})$, close to solar, populated by stars of type CEMP-s or CEMP-rs are members of binary systems whose carbon has been transferred by the more evolved companion during its AGB phase. This is supported by radial velocity measurements (Starkenburg et al., 2014; Lucatello et al., 2005).

- Those CEMP stars with carbon abundances roughly one dex below the solar carbon abundance are considered to have formed with their enhanced carbon abundance from gas enriched by nucleosynthesis of a few supernovae. We expect these stars to be CEMP-no. Unfortunately, it is very difficult (if not impossible) to detect heavy elements in these stars to be sure of this hypothesis.

The result is shown in Fig. 4 where we selected a range of iron abundances where the stars belonging to the two regions are comparable in number.

\section{Molecular bands}

We are investing considerable effort to try to model the molecular bands we use to derive the 


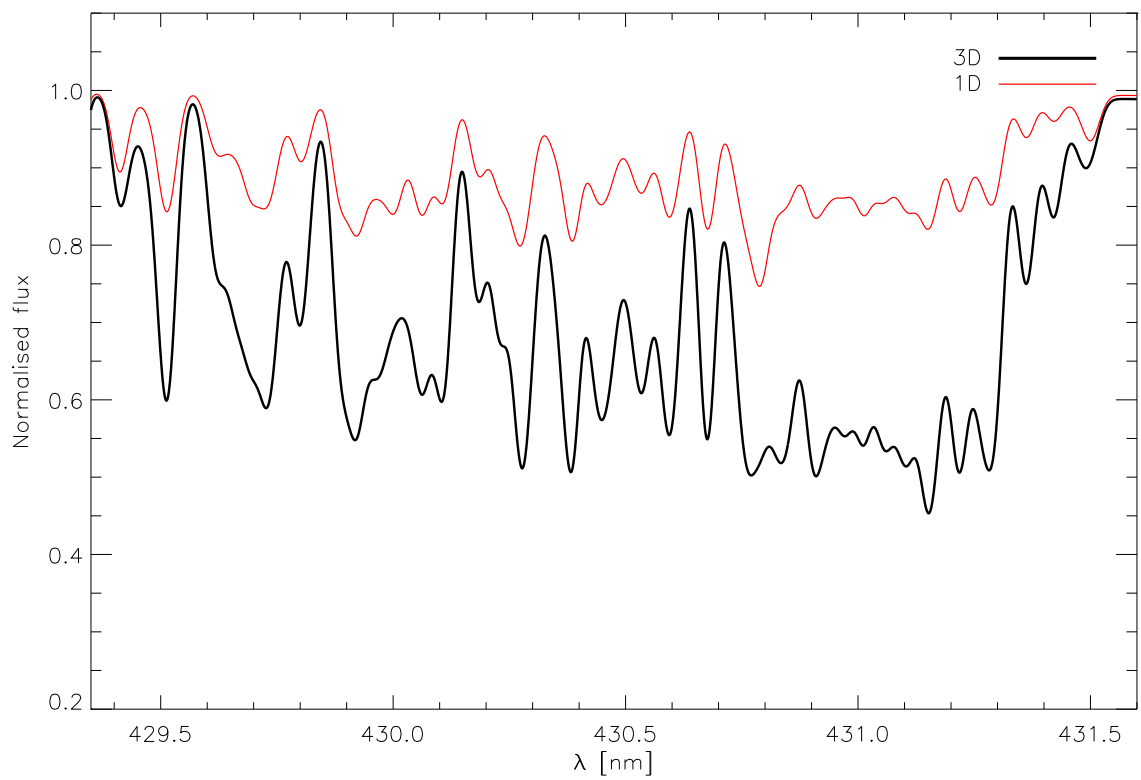

Figure 5: An example of the 3D and 1D synthesis of the G-band for a CEMP star. The 3D (black line) and 1D (red line) synthetic G-band heads for a CEMP star with $\mathrm{T}_{\text {eff }}=6300 \mathrm{~K}, \log \mathrm{g}=4.0,[\mathrm{Fe} / \mathrm{H}]=-3.0$ and $\mathrm{A}(\mathrm{C})=7.50$ dex are plotted.

abundances of the light elements carbon and nitrogen in the spectra of EMP stars as realistically as possible. We know that molecular lines form relatively high in the stellar photosphere and we also know that in these external layers the three-dimensional (3D) hydrodynamical models of EMP stars show a strong cooling, not seen in traditional one-dimensional (1D) models (see e.g. Bonifacio et al., 2013, and references therein). This cooling affects the molecular formation and as a consequence, the strength of the synthetic molecular lines that become stronger in $3 \mathrm{D}$. The resultant abundance derived from the molecular bands under a 3D paradigm is considerably smaller than under 1D, which is illustrated in Fig. 5.

One of the problems is that the molecular bands observable in EMP stars (e.g. the G-band at $430 \mathrm{~nm}$ ) are composed of hundreds of molecular lines and several metallic lines. The computation time to synthesise the complete G-band from a 3D model is too large. For a preliminary study, the G-band was computed for a carbon-enhanced metal-poor turn-off star. We parallelised the computation over eleven wavelength intervals and over twenty snapshots. We selected a region within the G-band that illustrated the 3D-1D line strength deviations well (shown in Fig. 5). We are currently working on the performance of the synthesis code to expedite synthesis production.

\section{References}

Andrievsky, S. M., Spite, F., Korotin, S. A., et al. 2011, A\&A, 530, A105

Bonifacio, P., Sbordone, L., Caffau, E., et al. 2012, A\&A, 542, A87

Bonifacio, P., Caffau, E., Ludwig, H.-G., et al. 2013, Memorie della Societa Astronomica Italiana Supplementi, 24, 138 
Bonifacio, P., et al. 2015, submitted to A\&A

Bromm, V., \& Loeb, A. 2003, Nature, 425, 812

Bromm, V. 2013, Reports on Progress in Physics, 76, 112901

Caffau, E., Bonifacio, P., François, P., et al. 2011a, A\&A, 534, A4

Caffau, E., Bonifacio, P., François, P., et al. 2011b, Nature, 477, 67

Caffau, E., Bonifacio, P., Sbordone, L., et al. 2013, A\&A, 560, A71

Christlieb, N., Bessell, M. S., Beers, T. C., et al. 2002, Nature, 419, 904

Christlieb, N., Gustafsson, B., Korn, A. J., et al. 2004, ApJ, 603, 708

Clark, P. C., Glover, S. C. O., Smith, R. J., et al. 2011, Science, 331, 1040

Dawson, K. S., Schlegel, D. J., Ahn, C. P., et al. 2013, AJ, 145, 10

Dopcke, G., Glover, S. C. O., Clark, P. C., \& Klessen, R. S. 2011, ApJ, 729, L3

Dopcke, G., Glover, S. C. O., Clark, P. C., \& Klessen, R. S. 2013, ApJ, 766, 103

Frebel, A., Aoki, W., Christlieb, N., et al. 2005, Nature, 434, 871

Glover, S. 2013, Astrophysics and Space Science Library, 396, 103

Hansen, C. J., Bergemann, M., Cescutti, G., et al. 2013, A\&A, 551, AA57

Hansen, T., Hansen, C. J., Christlieb, N., et al. 2014, ApJ, 787, 162

Keller, S. C., Bessell, M. S., Frebel, A., et al. 2014, Nature, 506, 463

Limongi, M., \& Chieffi, A. 2006, ApJ, 647, 483

Lucatello, S., Tsangarides, S., Beers, T. C., et al. 2005, ApJ, 625, 825

Masseron, T., Johnson, J. A., Plez, B., et al. 2010, A\&A, 509, A93

Norris, J. E., Christlieb, N., Korn, A. J., et al. 2007, ApJ, 670, 774

Omukai, K., Schneider, R., \& Haiman, Z. 2008, ApJ, 686, 801

Schneider, R., Omukai, K., Inoue, A. K., \& Ferrara, A. 2006, MNRAS, 369, 1437

Schneider, R., Omukai, K., Bianchi, S., \& Valiante, R. 2012, MNRAS, 419, 1566

Schörck, T., et al. 2009, A\&A, 507, 817

Spite, M., Caffau, E., Bonifacio, P., et al. 2013, A\&A, 552, A107

Starkenburg, E., Shetrone, M. D., McConnachie, A. W., \& Venn, K. A. 2014, MNRAS, 441, 121

Yanny, B., Rockosi, C., Newberg, H. J., et al. 2009, AJ, 137, 4377

York, D. G., et al. 2000, AJ, 120, 1579 